\title{
Electron heat flow due to magnetic field fluctuations
}

\author{
Journal-ref: Plasma Phys. Control. Fusion 58, 042001 (2016) \\ Jeong-Young Ji, ${ }^{1, *}$ Gunyoung Park, ${ }^{2}$ Sung Sik Kim, ${ }^{2}$ and Eric D. Held ${ }^{1}$ \\ ${ }^{1}$ Department of Physics, Utah State University, Logan, Utah 84322 \\ ${ }^{2}$ National Fusion Research Institute, Daejeon 305-333, Korea
}

(Dated: January 7, 2016)

\begin{abstract}
Radial heat transport induced by magnetic field line fluctuations is obtained from the integral parallel heat flow closure for arbitrary collisionality. The parallel heat flow and its radial component are computed for a single harmonic sinusoidal field line perturbation. In the collisional and collisionless limits, averaging the heat flow over an unperturbed surface yields Rechester-Rosenbluth like formulae with quantitative factors. The single harmonic result is generalized to multiple harmonics given a spectrum of small magnetic perturbations. In the collisionless limit, the heat and particle transport relations are also derived.
\end{abstract}

PACS numbers: 52.25.Fi,52.55.Dy 
Magnetic field line fluctuations can be generated by edge resonant magnetic perturbations (RMPs) [1], uncorrected ripple effects [2], instabilities [3], etc. For a stochastic magnetic field produced by overlapping magnetic islands [4], the radial heat transport, $h_{r}=-n \chi_{\mathrm{RR}} d T / d r$, has been estimated from a random walk process by Rechester and Rosenbluth (RR) [5]. The thermal diffusivity $\chi_{\mathrm{RR}}$ is given by

$$
\chi_{\mathrm{RR}}=v_{T} \pi L_{\mathrm{eff}} b_{\delta}^{2}
$$

where $n$ is the electron density, $T$ is the temperature, $v_{T}=\sqrt{2 T / m}$ is the thermal speed, $m$ is the electron mass, $L_{\text {eff }}$ is the effective autocorrelation length, and $b_{\delta}=B_{\delta} / B_{0}$ is the ratio of the radial fluctuation amplitude to the unperturbed magnetic field strength. The heat transport in a stochastic field has been investigated in recent experiments [6 8] and numerical simulations [9, 10]. Due to toroidal flow screening [11, 12], the fluctuating field could be magnetic flutter [13] with no island overlap. The particle and heat flows due to magnetic flutter in cylindrical [14, 15] and toroidal [15] geometry have been studied by Callen et al [16]. In a stochastic magnetic field, methodologies for simulating a heat diffusion equation $\partial_{t} T+\nabla \cdot \mathbf{q}=0$ with a given heat flux $\mathbf{q}$ have been developed [17, 18].

Although a random walk process can be used to qualitatively estimate the (thermal) diffusivity, this approach does not provide an important quantitative factor. As shown in Ref. [6], $\chi_{\mathrm{RR}}$ agrees with measured and numerically simulated thermal diffusivities where the field stochasticity (quantified by Chirikov's parameter) is high. However, in accordance with the derivation, $\chi_{\mathrm{RR}}$ does not agree where the stochasticity is low. In practical applications, it is ambiguous to distinguish between high and low stochasticity. It is also demonstrated that the RR transport is incompatible with integral (nonlocal) closures [18, 19].

To obtain quantitative closures or transport, one should solve the kinetic equation or equivalently the general moment equations. Importantly, when obtaining closures one must specify which moment equations are to be closed [20,21]. One of mathematical difficulties in solving the kinetic equation is accurately evaluating the Coulomb (Fokker-Planck, Landau) collision operators. Krook and Lorentz type operators have been adopted as model collision operators in deriving magnetic-flutter-induced transport [14, 15]. A numerical model operator that simulates particle collisions with a random walk process along the magnetic field lines and jumps across the field lines at collision events has been used in Ref. [22].

In this work we employ the integral (nonlocal) heat flow closure [23] obtained from the 
general moment equations with accurate Coulomb collision operators [24] to compute thermal transport along a magnetic field line. Whether the field line is stochastic or magnetic flutter, excursions of field lines off unperturbed, usually isothermal, surfaces yields the radial component of the parallel heat flow vector. We calculate the radial heat flow for a sinusoidal field line and extend the result to a general field line represented by a Fourier series. For a stochastic field or experimental data, a Fourier spectrum instead of a Fourier series can be practically used. For temporal fluctuation of the field lines, a spatial average may replace the time average. When averaged over an unperturbed surface, our result includes the RR theory in the collisional and collisionless limits. We provide a simple universal formula for the radial heat transport for small magnetic field perturbations.

For arbitrary collisionality, closures for density, temperature, and the flow velocity equations have been obtained in Ref. [25]. Therein, the parallel heat flux density responding to a temperature gradient is

$$
h_{\|}(\ell)=-\frac{1}{2} v_{T}(\ell) T(\ell) \int d \ell^{\prime} K_{h h}\left(\eta-\eta^{\prime}\right) \frac{n}{T} \frac{d T}{d \ell^{\prime}},
$$

where $\eta=\eta(\ell), \eta^{\prime}=\eta\left(\ell^{\prime}\right), \eta(\ell)=\int_{0}^{\ell} d \ell / \lambda_{\mathrm{C}}$ is the normalized arclength, $\ell$ is the arclength along a field line, and $\lambda_{\mathrm{C}}=v_{T} \tau_{\text {ee }}$ is the collision length defined by the electron thermal speed times the electron-electron collision time. The kernel function

$$
K_{h h}(\eta)=\left(-6.87+5.32 e^{-0.17 \eta^{0.646}}\right) \ln \left(1-e^{-2.02 \eta^{0.417}}\right)
$$

results from fitting to the 6400 moment solution in the high to low collisionality regimes and to the asymptotic form in the collisionless limit.

For a sinusoidal drive, the integral in Eq. (2) yields a simple formula. Consider a temperature profile $T=T_{0}+\delta T$ with a sinusoidal fluctuation $\delta T=T_{\delta} \sin \left(2 \pi \ell / \lambda_{\ell}+\varphi_{0}\right)=$ $T_{\delta} \sin \left(k \eta+\varphi_{0}\right)$, where $k=2 \pi \lambda_{\mathrm{C}} / \lambda_{\ell}$ measures the inverse collisionality. Assuming $T_{0} \gg \delta T$ and $n_{0} \gg \delta n$ ( $n_{0}$ is the average density and $\delta n$ is the density fluctuation), the heat flow is given by

$$
h_{\|}(\ell)=-\frac{1}{2} \hat{h}(k) n_{0} v_{T} T_{\delta} \cos \left(\frac{2 \pi \ell}{\lambda_{\ell}}+\varphi_{0}\right) .
$$

The coefficient $\hat{h}(k)$ is obtained either analytically with the moment- and collisionlesssolution kernels, or numerically with the fitted kernel (3). It is fitted within $1.6 \%$ error to

$$
\hat{h}(k)=\left(2.03-\frac{5.67 k^{1.27}}{1+4.22 k^{1.59}}\right) \tanh (1.58 k) .
$$




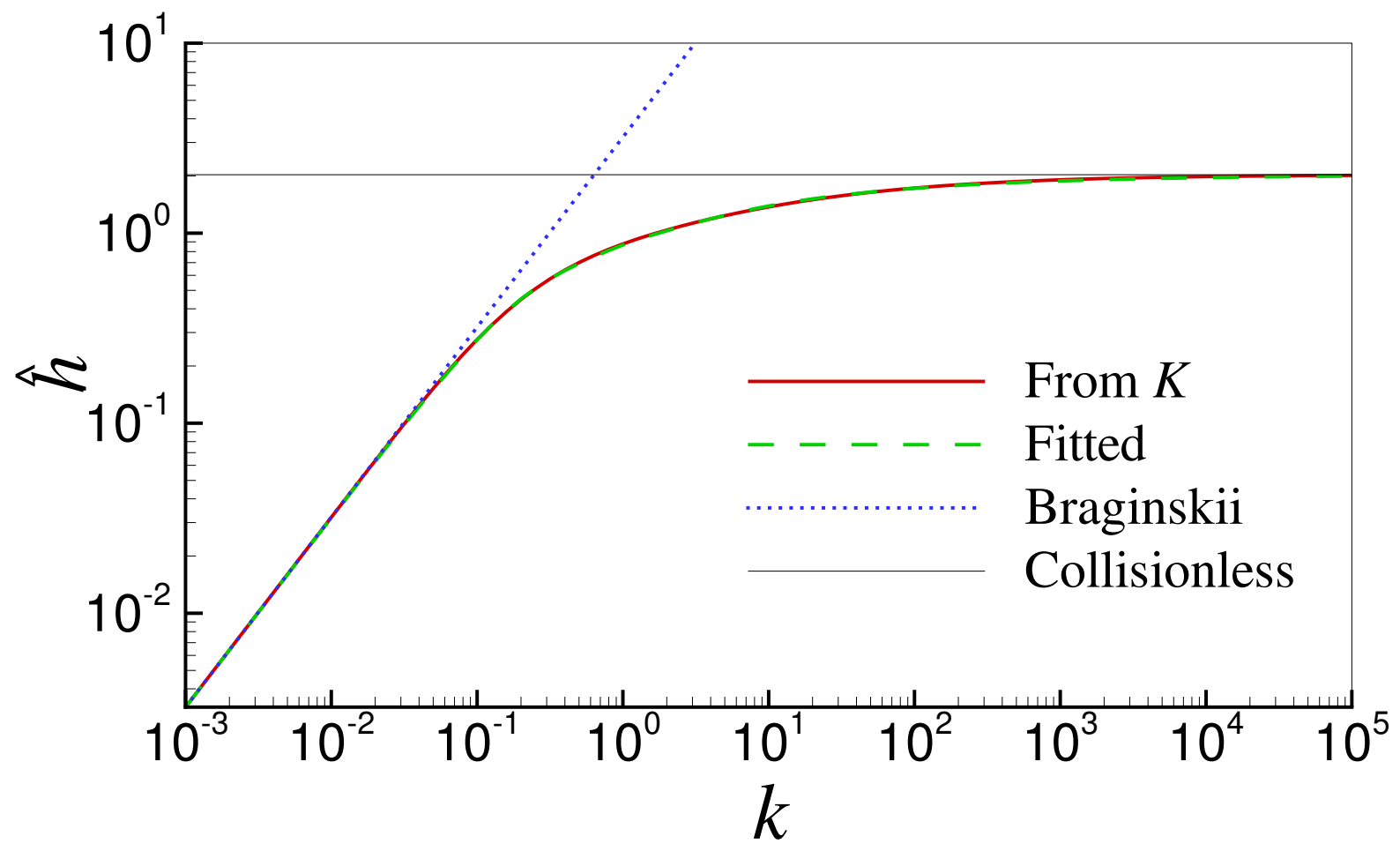

Figure 1: (Color online) Heat flow for a sinusoidal temperature profile from the kernel integral Eq. (2) (red, solid) and the fitted formula (5) (green, dashed). Braginskii's collisional (blue, dotted) and the collisionless (black, thin) limits are also shown.

This reproduces the collisional, $\hat{h} \rightarrow 4 \pi \chi_{\|} / v_{T} \lambda_{\ell} \approx 3.20 k$ as $k \rightarrow 0$, and collisionless, $\hat{h} \rightarrow$ $18 / 5 \sqrt{\pi}(\approx 2.03)$ as $k \rightarrow \infty$, limits (see Fig. 11).

To study parallel heat flow due to a fluctuating field line, we consider a sinusoidal field line trajectory

$$
r(z)=r_{0}+r_{\delta} \sin \frac{2 \pi}{\lambda} z
$$

where $r$ is the radial coordinate, $z$ is the coordinate along an unperturbed field line, $\lambda$ is the wavelength of the field line fluctuation measured in the $z$ direction, and $r_{\delta}$ is the amplitude of the fluctuation from the $r_{0}$ surface. Assuming that the unperturbed $r_{0}$ surface is isothermal, the temperature along the field line (므) is, to the linear $r_{\delta}$ order,

$$
T(z)=T_{0}\left(r_{0}\right)+T_{\delta} \sin \frac{2 \pi}{\lambda} z
$$

where

$$
T_{\delta}=\frac{d T_{0}}{d r} r_{\delta}
$$


The arclength coordinate $\ell$ along the field line is obtained from $\ell(z)=\int_{0}^{z} \sqrt{d z^{2}+d r^{2}}$. For Eq. (마),

$$
\ell(z)=\frac{\lambda}{2 \pi} \sqrt{1+a^{2}} E\left(\frac{2 \pi z}{\lambda}, \frac{a^{2}}{1+a^{2}}\right) \approx \alpha z
$$

where $a=2 \pi r_{\delta} / \lambda=b_{\delta}, E$ is the Legendre Elliptic integral of the second kind, Eq. (3-63) in Ref. [26], and $\alpha=\ell(\lambda) / \lambda$. For $a \lesssim 1$ (most cases), the approximation $\ell \approx \alpha z$ enables one to directly use Eq. (4) to write

$$
h_{\|}(z) \approx-\frac{1}{2} \hat{h}\left(\frac{k}{\alpha}\right) n_{0} v_{T} T_{\delta} \cos \frac{2 \pi z}{\lambda} .
$$

The error of this approximate heat flow is ignorable for most $a$ and $k$. Consider evaluating Eq. (9) at $z=0$. For the extreme case $a=1(\alpha \approx 1.2)$, the error is $16 \%$ in the collisional limit $(k \rightarrow 0)$ and decreases as $k$ increases, less than $10 \%$ error when $k \gtrsim 1$. For $a=$ $0.5(\alpha \approx 1.06)$, the error is $5.5 \%$ in the collisional limit and less than $3.4 \%$ when $k \gtrsim 1$. For $a=0.1(\alpha \approx 1.003)$, the error is less than $0.3 \%$. Therefore, for $a \ll 1(\alpha \approx 1)$, the approximation is practically exact. The advantage of this arclength approximation is that we can use the explicit formula (9) without performing the numerical integration. Using Eq. (9) also facilitates computing radial heat flows on a general field line for a given Fourier series or a spectrum of fluctuations.

Next we compute the radial heat flow across the unperturbed $r_{0}$ surface. For field lines penetrating the $r_{0}$ (isothermal) surface obliquely, the radial component of the parallel heat flow (9) is given by $h_{r}=(d r / d \ell) h_{\|}$. For a single harmonic (66), $d r / d \ell=$ $a \cos \varphi / \sqrt{1+a^{2} \cos ^{2} \varphi}$ where $\varphi=2 \pi z / \lambda$, and the radial heat flow at a local point is

$$
h_{r}(z)=-\frac{1}{2} \hat{h}\left(\frac{k}{\alpha}\right) n_{0} v_{T} T_{\delta} a \gamma(\varphi),
$$

where $\gamma(\varphi)=\cos ^{2} \varphi / \sqrt{1+a^{2} \cos ^{2} \varphi}$. When the field line fluctuation occurs also in the binormal direction $(\hat{r} \times \hat{z}$, a hat denoting a unit vector $)$, the elongation of the arclength $\ell$ will cause the increase of $\alpha$ and the decrease of $\gamma$, which will reduce the radial heat flux.

If the field line is static with no heating sources, the displaced surface will become isothermal due to thermal relaxation driven by the radial heat transport (10) and hence the transport is transient. In realistic situations, it is natural to consider fluctuating field lines and/or heating sources. In either case, Eq. (10) provides the heat flow closure/transport for given (transient) temperature and magnetic field profiles. While Eq. (10) describes the radial heat flow for a static field at a local point, the surface average of $h_{r}(z)$ is useful for studying heat 
confinement even when the field line is fluctuating in time. This surface average may replace the time average.

In slab or cylindrical geometry, the average is taken over one wavelength

$$
\left\langle h_{r}\right\rangle=\frac{1}{2 \pi} \int_{0}^{2 \pi} h_{r} d \varphi=-\frac{1}{2} \hat{h}\left(\frac{k}{\alpha}\right) n_{0} v_{T} T_{\delta} a\langle\gamma\rangle,
$$

where $\langle\gamma\rangle$ is between $0.38(a=1)$ and $1 / 2(a=0)$. In toroidal geometry, one needs a Jacobian for the flux surface average. In a wide range of applications we note that $b_{\delta} \ll 1$, e.g. $b_{\delta} \sim 10^{-4}$ for external RMPs. Using Eqs. (8) $, a=b_{\delta}, \alpha=1$, and $\langle\gamma\rangle=1 / 2$, we rewrite Eq. (11)

$$
\left\langle h_{r}\right\rangle=-\frac{1}{8 \pi} n_{0} v_{T} \hat{h}(k) \lambda b_{\delta}^{2} \frac{d T_{0}}{d r} .
$$

The effective thermal diffusivity for radial heat transport is

$$
\chi_{\mathrm{eff}}=\frac{1}{8 \pi} v_{T} \hat{h}(k) \lambda b_{\delta}^{2}
$$

Comparing with Eq. (1) for a stochastic field, the effective autocorrelation length is

$$
L_{\mathrm{eff}}=\frac{1}{8 \pi^{2}} \hat{h}(k) \lambda
$$

At this point it is interesting to compare Eq. (13) with the RR theory. Our theory may not describe the RR mechanism that is based on the diffusion of an area perpendicular to a stochastic magnetic field due to the Lyapunov divergence. In the both theories, however, the field line excursion off the isothermal surface result in radial heat flow due to parallel heat flow along the deviated field line. In computing the radial heat flow, the RR theory computes the field line deviation to estimate the qualitative radial diffusivity while our theory directly computes the radial component of the quantitative parallel heat flow. Of course, a stochastic field can not be represented by a single harmonic or a Fourier series of a perturbed field. However, a stochastic field can be described mathematically using a Fourier transform and measured experimentally (see Fig. 6 of Ref. [27]). Furthermore, one can use a single harmonic formula as an approximation by properly choosing the major mode with a proper weight factor. For comparison, we interpret $\lambda$ and $b_{\delta}$ in Eq. (13) as the effective wavelength and amplitude of the representative mode of perturbation spectrum. Accurate formula for a stochastic field will be obtained by adapting a Fourier series result to a Fourier spectrum. 
In the collisionless limit, $L_{\text {eff }} \approx \lambda / 4 \pi^{2}$ (using $\left.\hat{h} \approx 2\right)$ in this work while $L_{\text {eff }}=R(2 \pi R$ is the period of the system in the $z$ direction) in the RR theory [5] or $L_{\text {eff }}=q R / 2$ ( $q$ is the safety factor and $R$ is the major radius) in Ref. [10]. The ratio $\chi_{\text {eff }} / \chi_{\mathrm{RR}} \approx \lambda / 4 \pi^{2} R$ or $\chi_{\text {eff }} / \chi_{\text {Ref },[10]} \approx$ $\lambda / 2 \pi^{2} q R$ may possibly explain the large discrepancies observed in simulations [9, 10] and experiments [6, 27]. In Ref. [6], although the discrepancy is attributed to low stochasticity, the RR theory has a tendency to overestimate the experimental measurement even where stochasticity is high. In the collisional limit, $L_{\mathrm{eff}} \approx \chi_{\|} / 2 \pi v_{T}$. A smooth transition between the two regimes, $L_{\mathrm{eff}}^{-1}=L_{\mathrm{ac}}^{-1}+\lambda_{\mathrm{C}}^{-1}$ ( $L_{\mathrm{ac}}$ is the autocorrelation length), is suggested in Ref. [6]. Since Eq. (14) is valid for arbitrary collisionality, it naturally provides a similar smooth transition with precise numerical factors.

Now we consider a general field line perturbation expanded as a Fourier series

$$
r=r_{0}+\sum_{i} \delta r_{i}
$$

with

$$
\delta r_{i}=r_{\delta, i}^{\mathrm{c}} \cos \varphi_{i}+r_{\delta, i}^{\mathrm{s}} \sin \varphi_{i}
$$

where $\varphi_{i}=2 \pi z / \lambda_{i}$. The temperature along the field line is

$$
T=T_{0}+\frac{d T_{0}}{d r} \sum_{i} \delta r_{i}
$$

Assuming $b_{i}^{\mathrm{c}, \mathrm{s}}\left(=B_{\delta, i}^{\mathrm{c}, \mathrm{s}} / B_{0}=2 \pi r_{\delta, i}^{\mathrm{c}, \mathrm{s}} / \lambda_{i}\right) \ll 1$, the arclength of the perturbed field line is approximated by $z$. Then the parallel heat flow along the fluctuating field line is given by

$$
h_{\|}(z)=-\frac{1}{2} n_{0} v_{T} \frac{d T_{0}}{d r} \sum_{i} \hat{h}\left(k_{i}\right) \delta r_{i}^{\prime}
$$

with

$$
\delta r_{i}^{\prime} \equiv \frac{d}{d \varphi_{i}} \delta r_{i}=-r_{\delta, i}^{\mathrm{c}} \sin \varphi_{i}+r_{\delta, i}^{\mathrm{s}} \cos \varphi_{i}
$$

where $k_{i}=2 \pi \lambda_{\mathrm{C}} / \lambda_{i}$. Note in Eq. (18) that the roots of $d(\delta r) / d z=0\left(\right.$ or $\left.\sum_{i} 2 \pi \delta r_{i}^{\prime} / \lambda_{i}=0\right)$ do not agree with the roots of $\sum_{i} \hat{h}\left(k_{i}\right) \delta r_{i}^{\prime}=0$ except when $\hat{h} \propto k_{i}$ in the collisional limit (see Fig. (10)). Therefore, $\sum_{i} \hat{h}\left(k_{i}\right) \delta r_{i}^{\prime}>0[<0]$ can happen when $\sum_{i}\left(2 \pi / \lambda_{i}\right) \delta r_{i}^{\prime}<0[>0]$, which explains the occasional reversed heat flows (from low to high temperature) reported in Fig. 10 of Ref. [28]. Also $h_{\|} \neq 0$ happens when the field line is aligned along the unperturbed surface $\left(\sum_{i} 2 \pi \delta r_{i}^{\prime} / \lambda_{i}=0\right)$. Similar behavior, $h_{\|} \neq 0$ with $\partial_{\|} T=0$, is shown in Figs. 4 and 
6 of Ref. [23]. In general, the temperature gradient at a local point is not solely responsible for heat transport at the point. Such is the nature of integral (nonlocal) closures.

With the approximation $d r / d \ell \approx d r / d z\left(b_{r} \ll 1\right)$, the radial heat flow is

$$
h_{r}(z)= \pm \frac{d r}{d z} h_{\|}(z)
$$

where the + sign is for normal flows (from high to low temperature) and - is for reversed flows. When the reversed heat flows are ignorable, the radial component can be averaged to yield

$$
\left\langle h_{r}\right\rangle=-\frac{1}{8 \pi} n_{0} v_{T} \sum_{i} \hat{h}\left(k_{i}\right) \lambda_{i}\left[\left(b_{i}^{\mathrm{c}}\right)^{2}+\left(b_{i}^{\mathrm{s}}\right)^{2}\right] \frac{d T_{0}}{d r},
$$

for small fluctuations of multiple harmonics. Eq. (21) can be used when the field line is described by a spectrum of magnetic perturbations [27] by replacing $\sum_{i} \rightarrow \int d i$.

Eq. (21) provides a unified radial heat transport due to a stochastic magnetic field and magnetic flutter. Now we point out the limitations of this theory. First, the theory is valid in slab or cylindrical geometry. In toroidal geometry, the unperturbed field $B_{0}$ varies along the unperturbed field line and hence the results obtained here are approximate. Second, for large fluctuations in field lines, one should use Eq. (2) and $h_{r}=(d r / d \ell) h_{\|}$. When a stochastic field line wanders ergodically through a large volume, $\left\langle h_{r}\right\rangle \neq 0$ is observed even where $d T_{0} / d r=0$ (see Fig. 12 of Ref. [19]). In Refs. [18, 19], the collisional and collisionless closures are used uniformly. It would be interesting, however, to use Eq. (2) with kernel (3) consistently with local temperature. Third, Eq. (2) is one aspect of closures for density, temperature, and flow velocity equations. A complete set of electron parallel closures [25] includes heat flow, viscosity, and friction force responding to $\partial_{\|} T$ (parallel temperature gradient), $V_{\text {ei\| }}$ (difference between electron and ion parallel flow velocities), and $W_{\|}$[parallel component of the rate of strain tensor, $\left.W_{\alpha \beta}=\partial_{\alpha} V_{\beta}+\partial_{\beta} V_{\alpha}-(2 / 3) \delta_{\alpha \beta} \nabla \cdot \mathbf{V}\right]$. The other closures can be obtained in a similar way to deriving Eq. (12) or (21) from Eq. (21). Note that the formalisim developed in this work is valid for ions with electron variables replaced by ion variables. Formulas replacing Eqs. (3) and (5) for ions will appear in future work.

Since measuring $W_{\|}$is impractical in experiments, relating particle and heat transport to density and temperature gradients may be more convenient for verifying the theory. The particle and heat transport can be obtained by using the results of Ref. [21] in the collisionless limit. Noting that Eq. (2) in that limit reproduces Eq. (12) of Ref. [21] and comparing with 
Eq. (12) $(\hat{h}=18 / 5 \sqrt{\pi})$, we translate Eqs. (18) and (19) of Ref. [21] into

$$
\begin{aligned}
& \left\langle u_{r}\right\rangle=\frac{v_{T} \lambda b_{\delta}^{2}}{4 \pi^{3 / 2}}\left(-\frac{5}{4 p_{0}} \frac{d p_{0}}{d r}-\frac{5 q}{4 T_{0}} \frac{d \phi_{0}}{d r}+\frac{1}{2 T_{0}} \frac{d T_{0}}{d r}\right), \\
& \left\langle h_{r}\right\rangle=\frac{v_{T} \lambda b_{\delta}^{2}}{4 \pi^{3 / 2}}\left(\frac{1}{2} \frac{d p_{0}}{d r}+\frac{1}{2} n_{0} q \frac{d \phi_{0}}{d r}-2 n_{0} \frac{d T_{0}}{d r}\right)
\end{aligned}
$$

for electrons and ions with $v_{T}$ being the electron and ion thermal speed, respectively, and so on. Here $q$ is the electric charge $\left(q=-e\right.$ for electrons and $q=Z e$ for ions), $p_{0}\left(=n_{0} T_{0}\right)$ is the pressure, $\phi_{0}$ is the electric potential. For multiple harmonics or a spectrum, the corresponding radial transport can be obtained from Eqs. (22) and (23) by replacing $\lambda b_{r}^{2} \rightarrow$ $\sum_{i} \lambda_{i}\left[\left(b_{i}^{\mathrm{c}}\right)^{2}+\left(b_{i}^{\mathrm{s}}\right)^{2}\right]$ as was done in Eqs. (12) and (21).

One of the authors (Ji) would like to thank Dr. Hogun Jhang for suggesting this work during a visit to NFRI and Dr. Diego del-Castillo-Negrete for explaining and discussing Fig. 4, Ref. [18] and Fig. 12, Ref. [19]. The research is supported by the U.S. DOE under grant nos. DE-FG02-04ER54746, DE-FC02-04ER54798 and DE-FC02-05ER54812 and by the World Class Institute (WCI) Program of the National Research Foundation of Korea (NRF) funded by the Ministry of Education, Science and Technology of Korea (MEST) (NRF Grant No. WCI 2009-001). This work is performed in conjunction with the Plasma Science and Innovation (PSI) center and the Center for Extended MHD Modeling (CEMM).

* Electronic address: j.ji@usu.edu

[1] T. E. Evans, R. A. Moyer, P. R. Thomas, J. G. Watkins, T. H. Osborne, J. A. Boedo, E. J. Doyle, M. E. Fenstermacher, K. H. Finken, R. J. Groebner, et al., Phys. Rev. Lett. 92, 235003 (2004).

[2] P. N. Yushmanov, in Reviews of Plasma Physics, edited by M. A. Leontovich (Consultants Bureau, New York, 1990), vol. 17, p. 117.

[3] A. B. Mikhailovskii, Electromagnetic Instabilities in an Inhomogeneous Plasma (IOP Publishing Ltd, New York, 1992).

[4] M. Rosenbluth, R. Sagdeev, J. Taylor, and G. Zaslavski, Nuclear Fusion 6, 297 (1966).

[5] A. B. Rechester and M. N. Rosenbluth, Phys. Rev. Lett. 40, 38 (1978).

[6] T. M. Biewer, C. B. Forest, J. K. Anderson, G. Fiksel, B. Hudson, S. C. Prager, J. S. Sarff, J. C. Wright, D. L. Brower, W. X. Ding, et al., Phys. Rev. Lett. 91, 045004 (2003). 
[7] T. Evans, M. Fenstermacher, R. Moyer, T. Osborne, J. Watkins, P. Gohil, I. Joseph, M. Schaffer, L. Baylor, M. Bécoulet, et al., Nuclear Fusion 48, 024002 (2008).

[8] O. Schmitz, T. E. Evans, M. E. Fenstermacher, E. A. Unterberg, M. E. Austin, B. D. Bray, N. H. Brooks, H. Frerichs, M. Groth, M. W. Jakubowski, et al. (the DIII-D and TEXTOR Research Teams), Phys. Rev. Lett. 103, 165005 (2009).

[9] G. Park, C. S. Chang, I. Joseph, and R. A. Moyer, Phys. Plasmas 17, 102503 (2010).

[10] I. Joseph, T. E. Evans, A. M. Runov, M. E. Fenstermacher, M. Groth, S. V. Kasilov, C. J. Lasnjer, R. A. Moyer, G. D. Porter, M. J. Schaffer, et al., Nucl. Fusion 48, 045009 (2008).

[11] R. Fitzpatrick, Phys. Plasmas 5, 3325 (1998).

[12] V. Izzo and I. Joseph, Nuclear Fusion 48, 115004 (2008).

[13] J. D. Callen, Phys. Rev. Lett. 39, 1540 (1977).

[14] J. Callen, A. Cole, C. Hegna, S. Mordijck, and R. Moyer, Nuclear Fusion 52, 114005 (2012).

[15] J. D. Callen, A. J. Cole, and C. C. Hegna, Phys. Plasmas 19, 112505 (2012).

[16] J. Callen, C. Hegna, and A. Cole, Nuclear Fusion 53, 113015 (2013).

[17] S. R. Hudson and J. Breslau, Phys. Rev. Lett. 100, 095001 (2008).

[18] D. del Castillo-Negrete and L. Chacón, Phys. Rev. Lett. 106, 195004 (2011).

[19] D. Blazevski and D. del Castillo-Negrete, Phys. Rev. E 87, 063106 (2013).

[20] R. D. Hazeltine, Phys. Plasmas 5, 3282 (1998).

[21] J.-Y. Ji, E. D. Held, and H. Jhang, Phys. Plasmas 20, 082121 (2013).

[22] S. S. Abdullaev, Phys. Plasmas 20, 082507 (2013).

[23] J.-Y. Ji, E. D. Held, and C. R. Sovinec, Phys. Plasmas 16, 022312 (2009).

[24] J.-Y. Ji and E. D. Held, Phys. Plasmas 13, 102103 (2006).

[25] J.-Y. Ji and E. D. Held, Phys. Plasmas 21, 122116 (2014).

[26] J. Mathews and R. L. Walker, Mathematical Mathods of Physics (Addison-Wesley Publishing Company, Inc., Redwood City, 1970), 2nd ed.

[27] T. E. Evans, R. A. Moyer, K. H. Burrell, M. E. Fenstermacher, I. Joseph, A. W. Leonard, T. H. Osborne, G. D. Porter, M. J. Schaffer, P. B. Snyder, et al., Nature Phys. 2, 419 (2006).

[28] E. D. Held, J. D. Callen, C. C. Hegna, and C. R. Sovinec, Phys. Plasmas 8, 1171 (2001). 